Informationen der Arbeitsgemeinschaft Knochentumoren e. V.

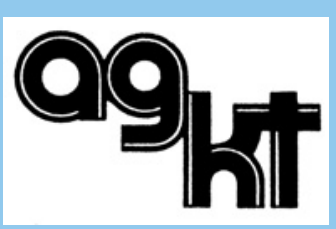

\title{
Tagungsbericht Herbsttagung der Arbeitsgemeinschaft Knochentumoren 2019
}

Im Herbst 2019 fand die 94. Tagung der AG Knochentumoren traditonsgemäß in München statt. In den Räumlichkeiten des Instituts für Pathologie an der TU München sorgten Prof. Wilko Weichert zusammen mit seinem Team um Frau PD Dr. Katja Specht und Frau PD Dr. Carolin Mogler für eine perfekt organisierte Veranstaltung in sehr angenehmer Atmosphäre.

Vor Beginn der eigentlichen Veranstaltung fand von 15.00 bis 16.00 Uhr die Vorstandssitzung statt, an die sich dann die Präsentation von Ad-hoc-Fällen anschloss (16.00 bis 18.00 Uhr), welche kurzfristig über das Sekretariat der AGKT (sekretariat@agkt.org) angemeldet werden oder spontan zur Sitzung mitgebracht werden können. Sie umfaßt Fälle, die akute diagnostische oder therapeutische Probleme beinhalten, zu denen Expertenmeinungen aus dem Kreis der AGKT (Orthopäden, Pathologen, Radiologen) erwünscht sind.

Vorgestellt wurde der Fall eines 15-jährigen Jungen, der seit Wochen über Schmerzen in der Fußsohle klagte. Radiologisch fanden sich Verkalkungen in den Weichteilen der Fußsohle in der Nähe von MFK4. Initial wurde der Verdacht auf ein Osteosarkom geäußert. Bei nachgewiesener USP6-Translokation und eindeutiger Lage außerhalb des Knochens konnte die Läsion als juxtakortikale Myositis ossificans eingeordnet werden.

Der zweite Fall betraf eine 40-jährige Frau mit seit zwei Monaten bestehenden Kniebeschwerden. Radiologisch fand sich eine Osteolyse (Lodwick II), die als ein maligner, offenbar unterschiedlich schnell gewachsener Tumor angesehen wurde. Differenzialdiagnostisch ergab die Biopsie histologisch den Verdacht auf ein benignes fibröses Histiozytom, eine aneurysmatische Knochenzyste oder einen Riesenzelltumor, wobei die AKZ (keine USP6-Translokation) und der Riesenzelltumor (negativ für H3F3A) ausgeschlossen werden konnten. Im Resektat fanden sich dann Areale, die aus atypischem Fettgewebe bestanden. Zusammen mit den BFH-ähnlichen Befunden und einer mdm2-Amplifikation wurde der Tumor als extrem seltenes intraossäres dedifferenziertes Liposarkom eingeordnet.

Da dieser Teil der Sitzung bereit um 17.00 Uhr beendet war, wurden die Präsentation und Diskussion eines Studienvorschlags zum Thema niedrig maligne intraossäre Osteosarkome vorgezogen, den Prof. Dr. Daniel Baumhoer aus Basel einbrachte.

Um 18 Uhr begannen die wissenschaftlichen Vorträge. Sie umfassten Themen aus der Radiologie und der Pathologie, die neuere Aspekte in der Diagnostik einiger gutartiger Knochenläsionen und neue Techniken in der Bildgebung beleuchteten, welche jeweils anschließend diskutiert wurden. Themen waren

\section{- Nicht ossifizierendes Knochenfibrom/} Benignes fibröses Histiozytom: ein Update (Prof. Dr. med. Daniel Baumhoer, Knochentumor-Referenzzentrum am Institut für Med. Genetik und Pathologie, Universitätsspital Basel)

\section{- MR-basierte CT-ähnliche Bildgebung} und simulierte konventionelle Radiografie (PD Dr. med. Alexandra S. Gersing Institut für diagnostische und interventionelle Radiologie Klinikum rechts der Isar, TU München)

- Neue Aspekte zu Riesenzelltumoren des Knochens (Prof. Dr. med. Thomas. Barth, Institut für Pathologie am Universitätsklinikum Ulm)

Am Ende seines Vortrags schlug Prof. Barth die Initiierung einer Verlaufsstudie zu Riesenzelltumoren vor, die mit Denosumab behandelt wurden.

(Die Zusammenfassung der Vorträge ist im Anschluss an den Tagungsbericht auf den Seiten 88-96 zu finden.)

\section{Mitgliederversammlung}

Am Samstagmorgen fand vor der wissenschaftlichen Falldiskussion die Mitgliederversammlung statt (8.30 bis 9.20 Uhr). Unter anderem wurde dem Antrag der Kollegen der TU München auf Anerkennung als Interdisziplinäres Zentrum für Knochentumoren stattgegeben, da alle satzungsgemäßen Voraussetzungen erfüllt sind (siehe Homepage: www.agkt.org). Somit ist die TU München neben Berlin, Basel, Bremen / Hannover, Münster und Ulm das 6. von der AGKT anerkannte Interdisziplinäre Zentrum für Knochentumoren.

Außerdem musste ein neuer Beisitzer gewählt werden, da die Amtszeit der Vorsitzenden, Frau Prof. Elisabeth Bruder/Basel, abgelaufen war und turnusgemäß Prof. Wörter/München als neuer Vorsitzender und Prof. Hardes / Essen als stv. Vorsitzender nachrückten. Einziger Kandidat war Prof. Barth / Ulm. Er wurde ohne Gegenstimmen bei einer Enthaltung gewählt, sodass wieder alle drei an der Knochentumordiagnostik und -therapie beteiligten Hauptdisziplinen (Radiologie, Orthopädie und Pathologie) im Vorstand vertreten sind.

Als neue Mitglieder wurden anschließend Frau PD Dr. med. Alexandra Gersing, wissenschaftliche Mitarbeiterin am Institut für diagnostische und interventionelle Radiologie am Klinikum rechts der Isar der Technischen Universität München (TUM), Dr. med. Alexander Klein, Facharzt für Orthopädie an der Orthopädischen Klinik und Poliklinik, Klinikum Großhadern, Klinikum der Universität München (LMU), und Frau PD Dr. med. Carolin Mogler, Geschäftsführende Oberärztin am Institut für Pathologie, Technische Universität München (TUM), ohne Gegenstimmen in die AGKT aufgenommen.

Anschließend hielt Prof. Remberger einen Nachruf auf unser am 23. März 2019 
verstorbenes Mitglied, Prof. Peter Meister aus München, indem er den Weg Peter Meisters in der Pathologie, sein Wirken und immerwährendes Interesse für das Fach nachzeichnete und seine Persönlichkeit würdigte.

\section{Wissenschaftliche Falldiskussion}

In der wissenschaftlichen Falldiskussion wurden neben einem Nachtrag (genetische Zusatzbefunde zu einem bereits vorgestellten Fall von progressiver ossärer Hyperplasie - $\mathrm{POH}$ ) zehn neue Fälle vorgestellt, die ausführlich diskutiert wurden. Betroffen waren jeweils fünf Frauen und Männer bei einer Altersverteilung von 18 bis 78 Jahren. 8-mal waren die langen Röhrenknochen befallen (5-mal Femur, je 1-mal Tibia, Fibula und Humerus), je 1-mal die Hand (Grundphalax D2) sowie die paraossären Weichteile des rechten Unterarms. Neben zwei gutartigen Läsionen (tumorähnliche Enthesiopathie bei Lithium-induzierter knochenbildender Psoriasis; ein gutartiger knochenbildender Tumor vom Typ eines Osteoblastoms mit schwieriger DD zum Osteosarkom) fanden sich acht maligne Tumoren. Drei davon gehörten in die erst in den letzten Jahren abgegrenzte Gruppe der undifferenzierten klein- und rundzelligen Sarkome (früher Ewing- und Ewing-like Sarkome), die nach der zugrunde liegenden Translokation in vier Gruppen eingeteilt werden können: die klassischen Ewing-Sarkome mit einer Fusion von Genen der FETund ETS- Familien (zu denen als häufigste die EWSR1-Fli1-Translokation gehört), die Rundzellsarkome mit einer EWSR1-non-ETS Fusion, wobei eine Fusion mit dem NFTAC2Gen hauptsächlich im Knochen vorkommt, während PATZ1-Fusionen vorwiegend in den Weichteilen beobachtet werden, sowie die CIC-rearrangierten Sarkome und die Sarkome mit genetischer BCOR-Alteration (Sbaraglia et al.: Ewing Sarcoma, Virchows Arch 2019 doi.org/10.1007/s00428-01902720-8; Grünewald et al.: Ewing sarcoma. Nat Rev Dis Primers 4, 5 [2018] doi:10.1038/ s41572-018-0003-x). Die übrigen fünf Tumoren umfassten ein in den paraossären Weichteilen gelegenes epitheloides Sarkom mit heterotoper Knochenbildung (DD extraskeletales Osteosarkom), ein regressiv verändertes Klarzellchondrosarkom, ein an der Hand ungewöhnliches Grad-2-Chondrosarkom, ein undifferenziertes intraossäres Sarkom und einen malignen Riesenzelltumor mit osteosarkomatöser Komponente unter Denosumab-Therapie.
Die Tagung wurde gegen 13.30 Uhr mit einem herzlichen Dankeschön an die Veranstalter für die hervorragende Organisation beendet.

Den Preis für die beste Fallpräsentation erhielten die Kollegen aus Budapest: Dr. János Kiss, Dr. András Rókusz, Dr. Imre Antal und Prof Dr. Miklós Szendrői, Orthopädische Klinik und Institut für Pathologie der Semmelweis-Universität Budapest (Fall 1121: "[Wahrscheinlich primär maligner] Riesenzelltumor mit Übergang in ein metastasierendes Osteosarkom unter Denosumab-Therapie»]).

\section{Zukünftige Tagungen}

Die Frühjahrstagung 2020 findet vom 27. bis 28. März 2020 in Ulm statt, die Herbsttagung 2020 am 16. und 17. Oktober wieder in München.

Falls Interesse besteht, als Gast an den Tagungen der AG Knochentumoren teilzunehmen, können weitere Informationen über das Sekretariat der AGKT in Basel (sek retariat@agkt.org) oder über die Homepage der AG Knochentumoren abgerufen werden(www.agkt.org). 